\title{
Improvement of Cardiac Function by Increasing Stimulus Strength During Left Ventricular Pacing in Cardiac Resynchronization Therapy
}

\author{
Kohei Ishibashi, ${ }^{1}$ MD, Takashi Kubo, ${ }^{1}$ MD, Hironori Kitabata, ${ }^{1}$ MD, Shigeho TaKarada, ${ }^{1}$ MD, \\ Kunihiro Shimamura, ${ }^{1}$ MD, Takashi Tanimoto, ${ }^{1}$ MD, Makoto OriI, ${ }^{1}$ MD, Yasutsugu Shiono, ${ }^{1}$ MD, \\ Takashi Yamano, ${ }^{1}$ MD, Yasushi Ino, ${ }^{1}$ MD, Tomoyuki Yamaguchi, ${ }^{1}$ MD, Kumiko Hirata,${ }^{1}$ MD, \\ Atsushi TanaKa, ${ }^{1} \mathrm{MD}$, Toshio Imanishi, ${ }^{1} \mathrm{MD}$, and Takashi AKasaKa, ${ }^{1} \mathrm{MD}$
}

\section{SUMMARY}

Cardiac resynchronization therapy (CRT) is an established therapy in patients with severe heart failure due to left ventricular (LV) dyssynchrony. Increasing stimulus strength (SS) of LV pacing could capture an enlarged myocardial area and provide rapid electrical conduction. The aim of the present study was to investigate whether increasing SS of LV pacing improves LV mechanical dyssynchrony and cardiac function in patients treated with CRT.

We enrolled 26 patients with CRT and changed the SS of LV pacing: $2.5 \mathrm{~V}$ (standard SS) and $5 \mathrm{~V}$ (high SS). Electrocardiography and echocardiography were performed to assess QRS duration, LV mechanical dyssynchrony, and cardiac function under each condition.

The QRS duration $(138.6 \pm 21.4 \mathrm{~ms}$ versus $126.8 \pm 23.1 \mathrm{~ms}, P<0.001)$, septal-posterior wall motion delay (126.5 $\pm 42.7 \mathrm{~ms}$ versus $111.4 \pm 55.3 \mathrm{~ms}, P=0.012)$, standard deviation of time from QRS $(69.6 \pm 21.8 \mathrm{~ms}$ versus $55.6 \pm 19.4$ $\mathrm{ms}, P<0.001), \mathrm{LV}$ ejection fraction ( $29.4 \pm 10.6 \%$ versus $33.4 \pm 11.6 \%, P=0.005)$, and $\mathrm{LV}$ stroke volume $(50.7 \pm 15.5$ $\mathrm{mL}$ versus $63.8 \pm 18.3 \mathrm{~mL}, P<0.001)$ improved significantly in high SS compared with standard SS.

Increasing SS of LV pacing in CRT improves LV mechanical dyssynchrony and cardiac function. The capture of an enlarged myocardial area by increasing SS of LV pacing might offer an acute hemodynamic benefit to patients treated with CRT. (Int Heart J 2015; 56: 62-66)

Key words: Left ventricular dyssynchrony, Heart failure

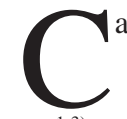
ardiac resynchronization therapy (CRT) has been proposed as a established therapy in patients with severe heart failure due to left ventricular (LV) dyssynchrony. ${ }^{1-3)}$ Several studies have demonstrated that CRT improves heart failure symptoms, quality of life, exercise capacity, and systolic LV performance.$^{1-6)}$ However, $20 \%$ to $30 \%$ of patients fail to obtain benefit from CRT. ${ }^{1-3)}$ The response to CRT is mainly associated with the LV lead location, which is often constrained by the anatomy of the coronary sinus and cardiac vein. Previous electrophysiology studies have shown that increasing stimulus strength (SS) of LV pacing captures an enlarged myocardial area and results in ventricular activation change. ${ }^{7)}$ If the capture of an enlarged myocardial area extends beyond a discrete region with conduction block, the increasing SS of LV pacing could provide more rapid electrical conduction in comparison with baseline. ${ }^{8-10)}$ Recent CRT studies have demonstrated that increasing SS of LV pacing reduces QRS duration on electrocardiograms (ECG) ${ }^{10,11)}$ The present study aimed to investigate whether increasing SS of LV pacing could improve LV mechanical dyssynchrony and cardiac function in patients treated with CRT.

\section{Methods}

Patient population: A total of 26 patients who underwent CRT with biventricular pacemakers or biventricular defibrillators according to clinical indications (New York Heart Association [NYHA] class III or IV heart failure, QRS > $120 \mathrm{~ms}$, LV ejection fraction $[\mathrm{LVEF}]<35 \%$, maximal medical therapy) were enrolled in the present study. We excluded the patients with extensive anterior myocardial infarction, LVEF $<10 \%$, and unsuccessful LV lead placement. The present study was approved by the Wakayama Medical University Ethics Committee, and all patients provided informed consent before participation.

Study protocol: The present study was performed at 6 months after CRT device implantation. The CRT device was programmed to DDD mode (lower rate limit, $60 \mathrm{bpm}$ ) to achieve atrial synchronous biventricular pacing. The atrio-ventricular

From the ${ }^{1}$ Department of Cardiovascular Medicine, Wakayama Medical University, Wakayama, Japan.

Address for correspondence: Takashi Kubo, MD, Department of Cardiovascular Medicine, Wakayama Medical University, 811-1, Kimiidera, Wakayama, 641-8510, Japan. E-mail: takakubo@wakayama-med.ac.jp

Received for publication April 24, 2014. Revised and accepted June 25, 2014.

Released in advance online on J-STAGE December 11, 2014.

All rights reserved by the International Heart Journal Association. 
delay and inter-ventricular delay were set at $120 \mathrm{~ms}$ and $0 \mathrm{~ms}$, respectively. The SS of LV pacing was changed into 3 conditions: 1) CRT-off, 2) $2.5 \mathrm{~V}$ (standard stimulus strength: standard SS), and 3) 5V (high stimulus strength: high SS). Electrocardiography and echocardiography were performed to assess QRS duration, QTc interval, LV mechanical dyssynchrony, and cardiac function under each condition (Figure 1).

Echocardiography: Standard 2D and tissue Doppler imaging was performed using a Vivid 7 echocardiograph (General Electric Medical Systems, Horten, Norway) equipped with a 3.5$\mathrm{MHz}$ phased array transducer. The images were acquired in a cine-loop format and stored digitally for offline analysis (EchoPAC, General Electric Medical Systems, Horten, Norway). LVEF, LV end-diastolic volume (LVEDV), and end-systolic volume (LVESV) were calculated using the Simpson biplane method according to the guidelines of the American Society of Echocardiography. ${ }^{12)}$ Velocity time integral (VTI) in the left ventricular outflow tract (LVOT) was calculated by Doppler echo, and stroke volume (SV) was calculated from VTI and LVOT diameter using the parasternal long-axis view. Echocardiographic assessments of LV mechanical dyssynchrony were as follows: 1) septal-posterior wall motion delay (SPWMD) measured by $\mathrm{M}$ mode of parasternal short-axis view; ${ }^{13)}$ 2) left ventricular pre-ejection interval (LPEI) defined as the time interval between the beginning of QRS and beginning of left ventricular ejection by Doppler echo; ${ }^{14)} 3$ ) interventricular mechanical delay (IVMD) defined as the difference between left and right ventricular pre-ejection intervals; ${ }^{14)}$ 4) LV filling ratio defined as LV filling time (LVFT) in relation to cardiac cycle length (CCL) as measured by transmitral Doppler echo (LVFT/ CCL) $;{ }^{14)}$ 5) septal to lateral delay (Ts-[1-s]), defined as the delay between time to peak systolic velocity at basal septal and basal lateral segments; ${ }^{15)}$ and 6 ) the standard deviation (SD) of time from QRS (Ts-SD), defined as the delay between time to peak systolic velocity in ejection phase for 12 left ventricular segments ( 6 basal and 6 middle). ${ }^{16,17)}$

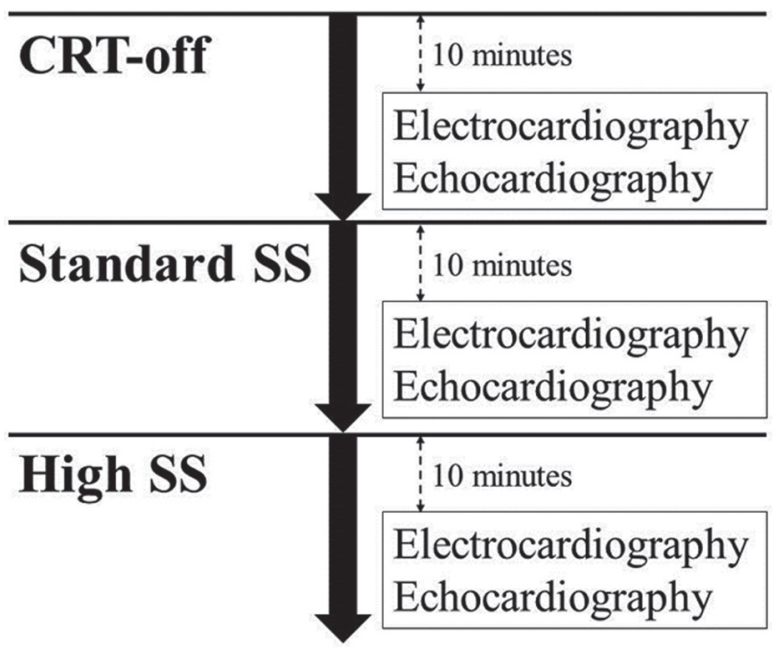

Figure 1. Study Protocol. Electrocardiography and echocardiography were performed under 3 conditions: SS of LV pacing $=0 \mathrm{~V}$ (CRT-off), 2.5 $\mathrm{V}$ (standard SS), and $5 \mathrm{~V}$ (high SS). A 10-minute interval was set between the change of SS and the examinations by electrocardiography and echocardiography. CRT indicates cardiac resynchronization therapy; LV, left ventricle; and SS, stimulus strength.
Statistical analysis: Statistical analysis was performed using SPSS version 11.0.1 (SPSS Inc., Chicago, IL). Results are expressed as the mean \pm SD for continuous variables. Categorical data are presented as numbers $(\%)$. Differences in continuous variables were assessed using the paired $t$-test. A $P$ value $<$ 0.05 was considered statistically significant.

\section{RESULTS}

Patient clinical characteristics are shown in Table I. There were 7 (27\%) patients with ischemic heart disease and 19 (73\%) patients with non-ischemic cardiomyopathy. The frequency of complete left bundle branch block (CLBBB), atrial fibrillation, and prior pacemaker implantation was 24 (92\%), 5 (19\%), and $4(15 \%)$, respectively.

The present study was performed at $6.6 \pm 1.9$ months after CRT device implantation. The LV lead was located in the lateral vein in $16(62 \%)$ patients, posterior-lateral vein in 3 $(12 \%)$, and anterior-lateral vein in $7(26 \%)$, but not in the anterior vein $(0 \%)$. The $\mathrm{LV}$ pacing threshold was $1.0 \pm 0.4 \mathrm{~V}$ (range, $0.5 \sim 1.8 \mathrm{~V}$ ) with a pulse width of $0.5 \mathrm{~ms}$. During standard SS and high SS, the mean pulse width of the LV pacing was $0.7 \pm 0.4 \mathrm{~ms}$ (range, $0.4 \sim 1.5 \mathrm{~ms}$ ). Phrenic nerve stimulation was observed in $2(8 \%)$ patients at high SS of LV pacing. All RV leads were located in the RV apex. The RV pacing threshold was $0.8 \pm 0.3 \mathrm{~V}$ (range, $0.3 \sim 1.5 \mathrm{~V}$ ) with a pulse width of $0.5 \mathrm{~ms}$. During examination, the mean SS and pulse width of RV pacing were $2.6 \pm 0.4 \mathrm{~V}$ (range, $2.0 \sim 3.5 \mathrm{~V}$ ) and $0.5 \pm 0.1 \mathrm{~ms}$ (range, $0.4 \sim 1.0 \mathrm{~ms}$ ), respectively.

ECG and echocardiography findings in CRT-off are shown in Table II. The QRS duration on ECG was $158.4 \pm$ $42.7 \mathrm{~ms}$. On echocardiography, SPWMD and LVEF were $196.2 \pm 100.4 \mathrm{~ms}$ and $24.9 \pm 7.1 \%$, respectively.

The changes in the ECG and echocardiography findings between standard SS and high SS are shown in Table III. In ECG, the QRS duration decreased significantly in high SS

Table I. Patient Clinical Characteristics

\begin{tabular}{lc}
\hline Variable & \\
\hline Number & 26 \\
Age, years & $67 \pm 13$ \\
Male & $20(77)$ \\
Ischemic heart disease & $7(27)$ \\
Non-ischemic cardiomyopathy & $19(73)$ \\
Dilated cardiomyopathy & $12(46)$ \\
Hypertrophic cardiomyopathy & $3(12)$ \\
Myocarditis & $2(8)$ \\
Cardiac sarcoidosis & $1(4)$ \\
Valuvular heart disease & $1(4)$ \\
CLBBB & $24(92)$ \\
Atrial fibrillation & $5(19)$ \\
Prior anti-brady pacemaker & $4(15)$ \\
NYHA class & \\
I & $3(12)$ \\
II & $3(12)$ \\
III & $20(76)$ \\
IV & $0(0)$ \\
\hline
\end{tabular}

Values are given as $n(\%)$ or mean \pm standard deviation. CLBBB indicates complete left bundle branch block; and NYHA, New York Heart Association. 
Table II. Electrocardiography and Echocardiography Findings in CRT-Off

\begin{tabular}{lc}
\hline Variable & \\
\hline Electrocardiography & \\
QRS duration, ms & $158.4 \pm 42.7$ \\
QTc interval, ms & $509 \pm 48$ \\
Echocardiography & \\
Mechanical dyssynchrony parameters & $196.2 \pm 100.4$ \\
SPWMD, ms & $142.2 \pm 52.4$ \\
LPEI, ms & $32.7 \pm 32.6$ \\
IVMD, ms & $46.7 \pm 12.3$ \\
LVFT/CCL, \% & $102.9 \pm 51.1$ \\
Ts-SD, ms & $32.8 \pm 123.0$ \\
Ts(l-s), ms & \\
Cardiac function parameters & $216.0 \pm 69.4$ \\
LVEDV, mL & $165.1 \pm 66.1$ \\
LVESV, mL & $24.9 \pm 7.1$ \\
LVEF, \% & $10.4 \pm 4.0$ \\
VTI in LVOT, cm & $42.3 \pm 14.7$ \\
SV, mL & $25.0 \pm 5.9$ \\
TR-PG, mmHg &
\end{tabular}

Values are given as mean \pm standard deviation. CCL indicates cardiac cycle length; IVMD, interventricular mechanical delay; LPEI, left ventricular pre-ejection interval; LVEDV, left ventricular end-diastolic volume; LVEF, left ventricular ejection fraction; LVESV, left ventricular end-systolic volume; LVFT, left ventricular filling time; LVOT, left ventricular outflow tract; SD, standard deviation; SS, stimulus strength; SPWMD, septalposterior wall motion delay; SV, stroke volume; TR-PG, tricuspid regurgitation-pressure gradient; Ts, time to peak systolic velocity; and VTI, velocity time integral.

Table III. Changes of Electrocardiography and Echocardiography Findings Between Standard SS and High SS

\begin{tabular}{lccr}
\hline & $\begin{array}{c}\text { Standard SS } \\
(2.5 \mathrm{~V})\end{array}$ & $\begin{array}{c}\text { High SS } \\
(5 \mathrm{~V})\end{array}$ & $P$ \\
\hline Electrocardiography & & & \\
QRS duration, ms & $138.6 \pm 21.4$ & $126.8 \pm 23.1$ & $<0.001$ \\
QTc interval, ms & $525 \pm 38$ & $536 \pm 43$ & 0.048 \\
Echocardiography & & & \\
Mechanical dyssynchrony parameters & & \\
SPWMD, ms & $126.5 \pm 42.7$ & $111.4 \pm 55.3$ & 0.012 \\
LPEI, ms & $147.6 \pm 36.4$ & $146.9 \pm 31.1$ & 0.155 \\
IVMD, ms & $27.3 \pm 17.5$ & $30.5 \pm 26.0$ & 0.413 \\
LVFT/CCL, \% & $50.7 \pm 7.9$ & $52.5 \pm 10.5$ & 0.391 \\
Ts-SD, ms & $69.6 \pm 21.8$ & $55.6 \pm 19.4$ & $<0.001$ \\
Ts(l-s), ms & $22.7 \pm 72.9$ & $15.8 \pm 69.1$ & 0.620 \\
Cardiac function parameters & & & \\
LVEDV, mL & $206.2 \pm 84.7$ & $201.9 \pm 82.2$ & 0.198 \\
LVESV, mL & $151.8 \pm 83.8$ & $144.3 \pm 80.5$ & 0.020 \\
LVEF, \% & $29.4 \pm 10.6$ & $33.4 \pm 11.6$ & 0.005 \\
VTI in LVOT, cm & $13.0 \pm 5.1$ & $17.1 \pm 7.4$ & $<0.001$ \\
SV, mL & $50.7 \pm 15.5$ & $63.8 \pm 18.3$ & $<0.001$ \\
TR-PG, mmHg & $23.2 \pm 5.5$ & $24.2 \pm 5.9$ & 0.187 \\
\hline
\end{tabular}

Values are given as mean \pm standard deviation. CCL indicates cardiac cycle length; IVMD, interventricular mechanical delay; LPEI, left ventricular pre-ejection interval; LVEDV, left ventricular end-diastolic volume; LVEF, left ventricular ejection fraction; LVESV, left ventricular end-systolic volume; LVFT, left ventricular filling time; LVOT, left ventricular outflow tract; SD, standard deviation; SS, stimulus strength; SPWMD, septalposterior wall motion delay; SV, stroke volume; TR-PG, tricuspid regurgitation-pressure gradient; Ts, time to peak systolic velocity; and VTI, velocity time integral.

compared with standard SS $(P<0.001)$. In echocardiography, the LV mechanical dyssynchrony parameters SPWMD $(P=$

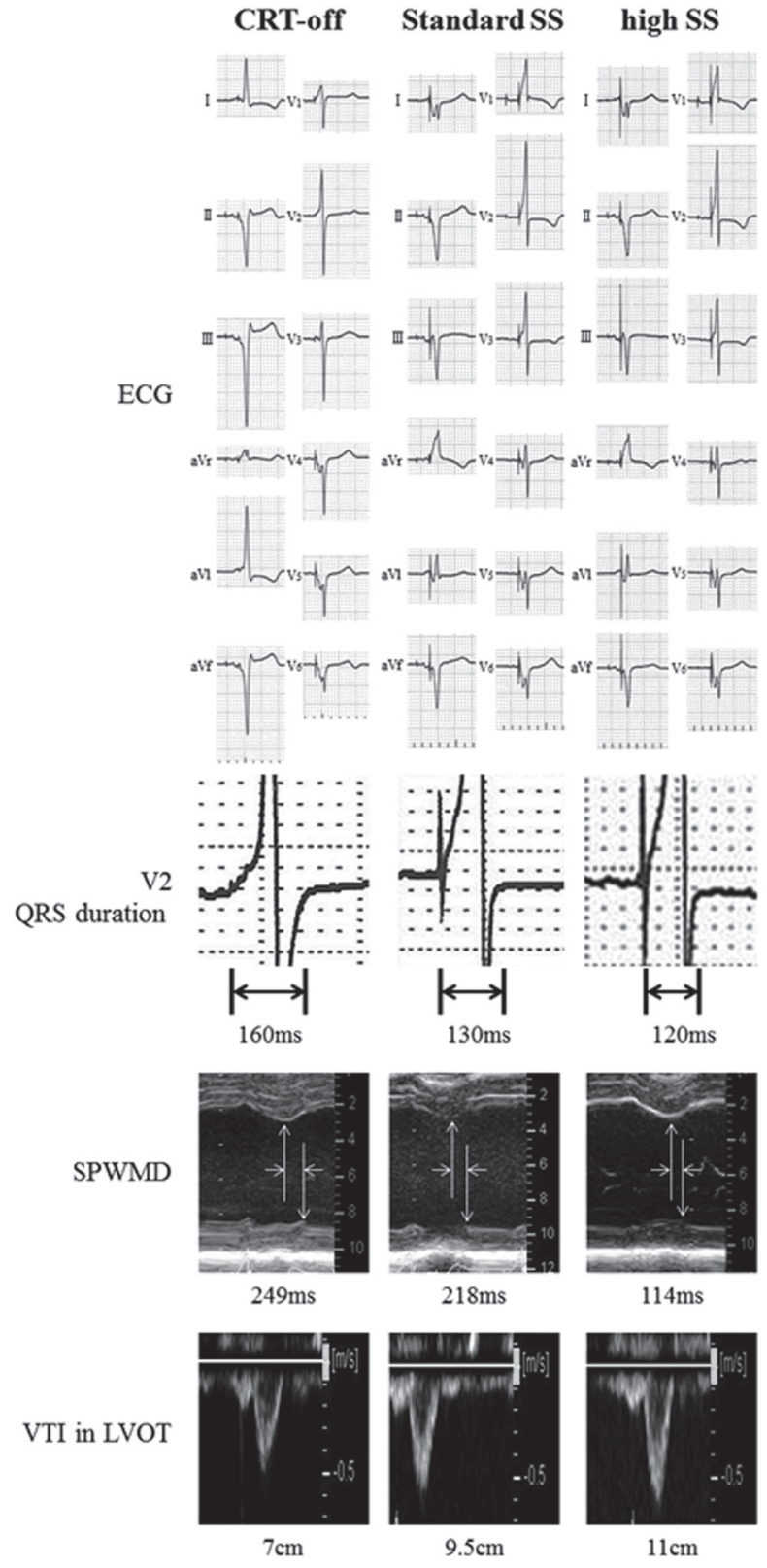

Figure 2. Representative case with an improvement of ECG and echocardiographic findings by increasing SS of LV pacing. The QRS duration on ECG and SPWMD and VTI in LVOT on echocardiography improved in high SS compared with standard SS. The right atrial and right ventricular pacing was performed even in CRF-off, because this case presented sinus bradycardia and complete atrioventricular block at baseline. CRT indicates cardiac resynchronization therapy; ECG, electrocardiogram; LV, left ventricle; LVOT, left ventricular outflow tract; SPWMD, septal-posterior wall motion delay; SS, stimulus strength; and VTI, velocity time integral.

0.012) and Ts-SD $(P<0.001)$ improved significantly in high SS compared with standard SS. Also, the cardiac function parameters LVESV $(P=0.020), \operatorname{LVEF}(P=0.005)$, VTI in LVOT $(P<0.001)$, and SV $(P<0.001)$ improved significantly in high SS compared with standard SS. A representative case is shown in Figure 2. 


\section{DisCUSSION}

The present study demonstrated that an increase in SS of LV pacing has the potential to improve LV mechanical dyssynchrony and cardiac systolic function in patients treated with CRT. To the best of our knowledge, the present study is the first to demonstrate the efficacy of high SS of LV pacing in CRT.

Pathologic condition of the LV is a major determinant of successful CRT. The scar tissue at the region of the LV lead inhibits effective pacing of myocardium, resulting in no change of QRS duration on the ECG. ${ }^{18,19)}$ Increasing SS of LV pacing in CRT captures an enlarged myocardial area and provides a pacing response beyond a discrete region with conduction block, which thereby leads to more rapid electrical conduction in comparison with baseline. ${ }^{7-10)}$ This effect appears prominently in low-amplitude areas of electroanatomic voltage maps, such as infarct borders. ${ }^{20)}$ Recently, Tedrow, et al demonstrated that increasing SS of LV pacing in CRT reduces inter-ventricular conduction time and QRS duration. ${ }^{10)}$ Moreover, the present study revealed that increasing SS of LV pacing in CRT provided not only reduction of QRS duration but also improvement of cardiac systolic function. Increasing SS of LV pacing in CRT may offer an acute hemodynamic benefit to CRT non-responders.

It is unknown whether QRS narrowing in CRT is associated with improvement of cardiac function. According to the recent reports of meta-analysis assessing the impact of QRS narrowing in CRT, 7 studies showed a good association between QRS narrowing and improvement of cardiac function, while 35 studies demonstrated that the association is slight or unknown. ${ }^{21,22)}$ Because wide QRS duration does not necessarily reflect the heterogeneous propagation pattern of LV activation and prolonged duration of mechanical activation, the association between these parameters might be unclear. In the present study, some patients showed improvements in LV mechanical dyssynchrony and cardiac systolic function without significant QRS narrowing by CRT with high SS. Therefore, increasing SS of LV pacing might be useful for patients with CRT regardless of their QRS duration.

Limitations: There are several limitations in our study. First, the area of scar tissue was not evaluated. If the area of scar tissue was broadly distributed, increasing SS of LV pacing cannot provide the pacing response beyond a discrete region with conduction block. Second, the present study assessed only LV mechanical dyssynchrony and cardiac function immediately after increasing SS of LV pacing in CRT. Therefore, long-term clinical outcome data by increasing SS of LV pacing in CRT is unknown. Third, there were 5 patients with atrial fibrillation. Since the ventricular rate was well controlled, adequate biventricular pacing was achieved in these patients. Fourth, several mechanical dyssynchrony parameters were improved by increasing SS in the present study, however, it is still unclear whether these parameters have important implications for response of CRT. Finally, increasing SS of LV pacing in CRT may not be practical in the clinical setting. High SS of LV pacing would involve a risk for phrenic nerve stimulation and might cause dramatic battery consumption. Multisite LV pacing can capture an enlarged myocardial area without phrenic nerve stimulation and may improve LV mechanical dyssynchrony and cardiac function similar to high SS of LV pacing.
Our study provides the basic data that supports the efficacy for capture of an enlarged myocardial area by LV pacing in CRT. Conclusions: Increasing SS of LV pacing in CRT improves LV mechanical dyssynchrony and cardiac function. The capture of an enlarged myocardial area by increasing SS of LV pacing might offer an acute hemodynamic benefit to patients treated with CRT.

\section{Disclosures}

All authors, do not have a financial interest/arrangement or affiliation with one or more organizations that could be perceived as a real or apparent conflict of interest in the context of the subject of this article.

\section{REFERENCES}

1. Abraham WT, Hayes DL. Cardiac resynchronization therapy for heart failure. Circulation 2003; 108: 2596-603. (Review)

2. Leclercq C, Kass DA. Retiming the failing heart: principles and current clinical status of cardiac resynchronization. J Am Coll Cardiol 2002; 39: 194-201. (Review)

3. Leclercq C, Hare JM. Ventricular resynchronization: current state of the art. Circulation 2004; 109: 296-9. (Review)

4. Cazeau S, Leclercq C, Lavergne T, et al. Effects of multisite biventricular pacing in patients with heart failure and intraventricular conduction delay. N Engl J Med 2001; 344: 873-80.

5. Abraham WT, Fisher WG, Smith AL, et al. Cardiac resynchronization in chronic heart failure. N Engl J Med 2002; 346: 1845-53.

6. Auricchio A, Stellbrink C, Sack S, et al. Long-term clinical effect of hemodynamically optimized cardiac resynchronization therapy in patients with heart failure and ventricular conduction delay. $\mathrm{J}$ Am Coll Cardiol 2002; 39: 2026-33.

7. Soejima K, Stevenson WG, Maisel WH, Sapp JL, Epstein LM. Electrically unexcitable scar mapping based on pacing threshold for identification of the reentry circuit isthmus: feasibility for guiding ventricular tachycardia ablation. Circulation 2002; 106: 167883.

8. Rickard J, Popovic Z, Verhaert D, et al. The QRS narrowing index predicts reverse left ventricular remodeling following cardiac resynchronization therapy. Pacing Clin Electrophysiol 2011; 34: 604-11.

9. Sauer WH, Sussman JS, Verdino RJ, Cooper JM. Increasing left ventricular pacing output decreases interventricular conduction time in patients with biventricular pacing systems. Pacing Clin Electrophysiol 2006; 29: 569-73.

10. Tedrow U, Maisel WH, Epstein LM, Soejima K, Stevenson WG. Feasibility of adjusting paced left ventricular activation by manipulating stimulus strength. J Am Coll Cardiol 2004; 44: 2249-52.

11. Theis C, Bavikati VV, Langberg JJ, Lloyd MS. The relationship of bipolar left ventricular pacing stimulus intensity to cardiac depolarization and repolarization in humans with cardiac resynchronization devices. J Cardiovasc Electrophysiol 2009; 20: 645-9.

12. Schiller NB, Shah PM, Crawford M, et al. Recommendations for quantitation of the left ventricle by two-dimensional echocardiography. American Society of Echocardiography Committee on Standards, Subcommittee on Quantitation of Two-Dimensional Echocardiograms. J Am Soc Echocardiogr 1989; 2: 358-67. (Review)

13. Pitzalis MV, Iacoviello M, Romito R, et al. Cardiac resynchronization therapy tailored by echocardiographic evaluation of ventricular asynchrony. J Am Coll Cardiol 2002; 40: 1615-22.

14. Cazeau S, Bordachar P, Jauvert G, et al. Echocardiographic modeling of cardiac dyssynchrony before and during multisite stimulation: a prospective study. Pacing Clin Electrophysiol 2003; 26: 
$137-43$.

15. Bax JJ, Marwick TH, Molhoek SG, et al. Left ventricular dyssynchrony predicts benefit of cardiac resynchronization therapy in patients with end-stage heart failure before pacemaker implantation. Am J Cardiol 2003; 92: 1238-40.

16. Yu CM, Fung WH, Lin H, Zhang Q, Sanderson JE, Lau CP. Predictors of left ventricular reverse remodeling after cardiac resynchronization therapy for heart failure secondary to idiopathic dilated or ischemic cardiomyopathy. Am J Cardiol 2003; 91: 684-8.

17. Yu CM, Fung JW, Zhang Q, et al. Tissue Doppler imaging is superior to strain rate imaging and postsystolic shortening on the prediction of reverse remodeling in both ischemic and nonischemic heart failure after cardiac resynchronization therapy. Circulation 2004; 110: 66-73.

18. Ypenburg C, Schalij MJ, Bleeker GB, et al. Impact of viability and scar tissue on response to cardiac resynchronization therapy in ischaemic heart failure patients. Eur Heart J 2007; 28: 33-41.

19. Bleeker GB, Kaandorp TA, Lamb HJ, et al. Effect of posterolateral scar tissue on clinical and echocardiographic improvement after cardiac resynchronization therapy. Circulation 2006; 113: 969-76.

20. Marchlinski FE, Callans DJ, Gottlieb CD, Zado E. Linear ablation lesions for control of unmappable ventricular tachycardia in patients with ischemic and nonischemic cardiomyopathy. Circulation 2000; 101: 1288-96.

21. Kashani A, Barold SS. Significance of QRS complex duration in patients with heart failure. J Am Coll Cardiol 2005; 46: 2183-92. (Review)

22. Hawkins NM, Petrie MC, MacDonald MR, Hogg KJ, McMurray JJ. Selecting patients for cardiac resynchronization therapy: electrical or mechanical dyssynchrony? Eur Heart J 2006; 27: 127081. (Review) 